\title{
Fabric beats in radar data across the NEGIS ice stream
}

- determining horizontal anisotropy in ice from co-polarized profiling data -

O. Eisen ${ }^{1}$, S. Franke1, D. Jansen ${ }^{1}$, J. Paden², R. Drews ${ }^{3}$, M. R. Ershadi ${ }^{3}$ D. Steinhage1, V. Helm¹, D. Lilien ${ }^{4}$, A. Grindsted ${ }^{4}$, C. Hvidberg ${ }^{4}$, A. Humbert ${ }^{1}$, M. Rückamp ${ }^{1}$, J. Yan ${ }^{5}$, I. Weikusat ${ }^{1}$, F. Wilhelms ${ }^{1}$, D. Dahl-Jensen ${ }^{4}$, H. Miller ${ }^{1}$

1 AWI, Bremerhaven, Germany, 2 CReSIS, Kansas, USA, 3 Uni Tübingen, Germany, 4 PICE, Uni Copenhagen, Denmark, 5 Univ Alabama, USA

$$
\text { \#vEGU21 }
$$




\section{NEGIS ice stream \& airborne survey बANI}

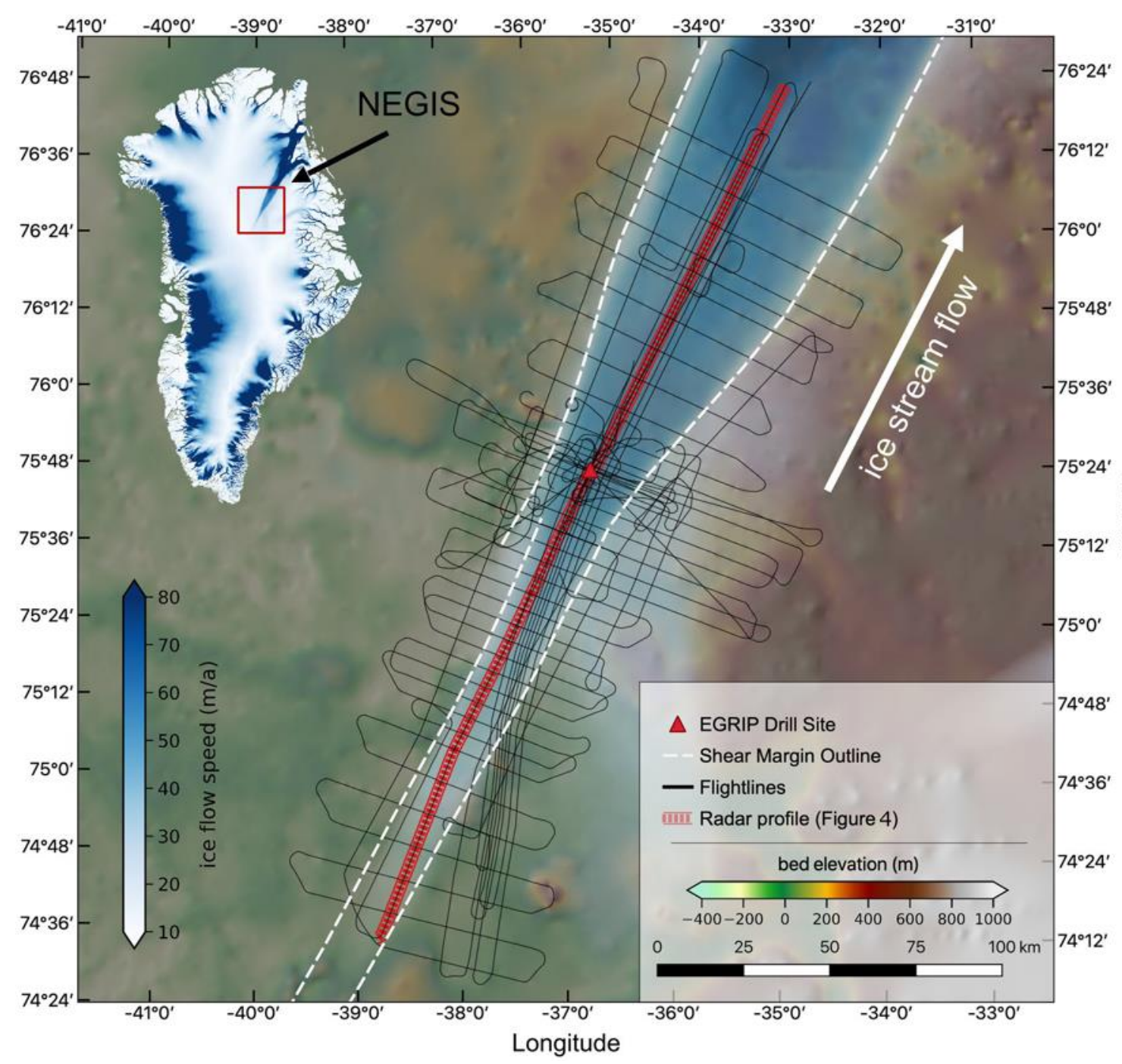

further details: Franke et al., JGR, 2020

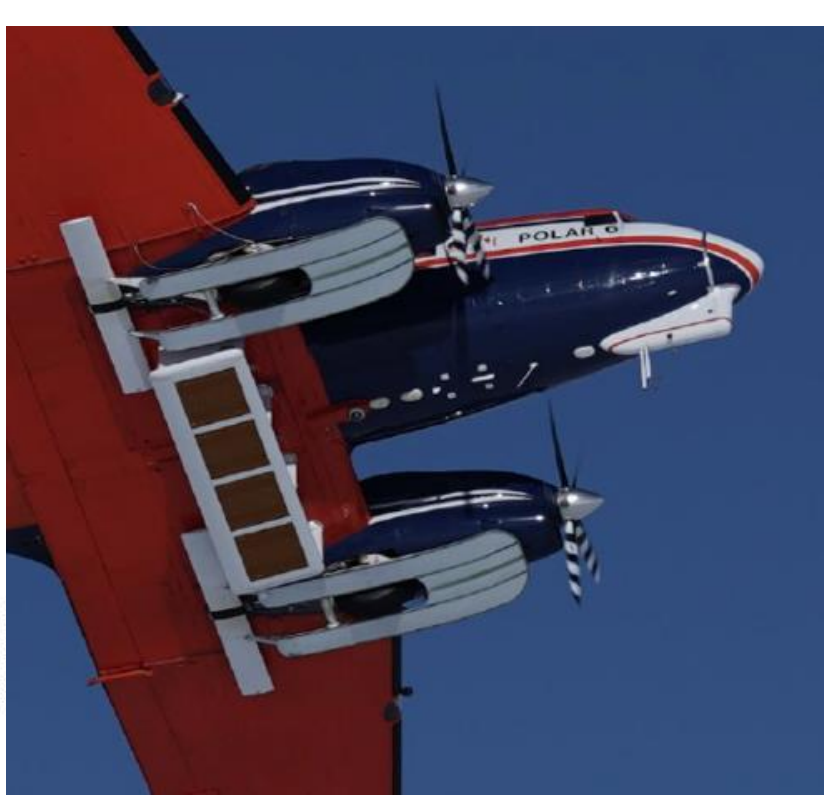

Survey: May 2018

Radar: AWI UWB

MCORDS5, 8 elements

Operation in narrow band mode: $180-210 \mathrm{MHz}$ 


\section{Across flow radargrams NEGIS}

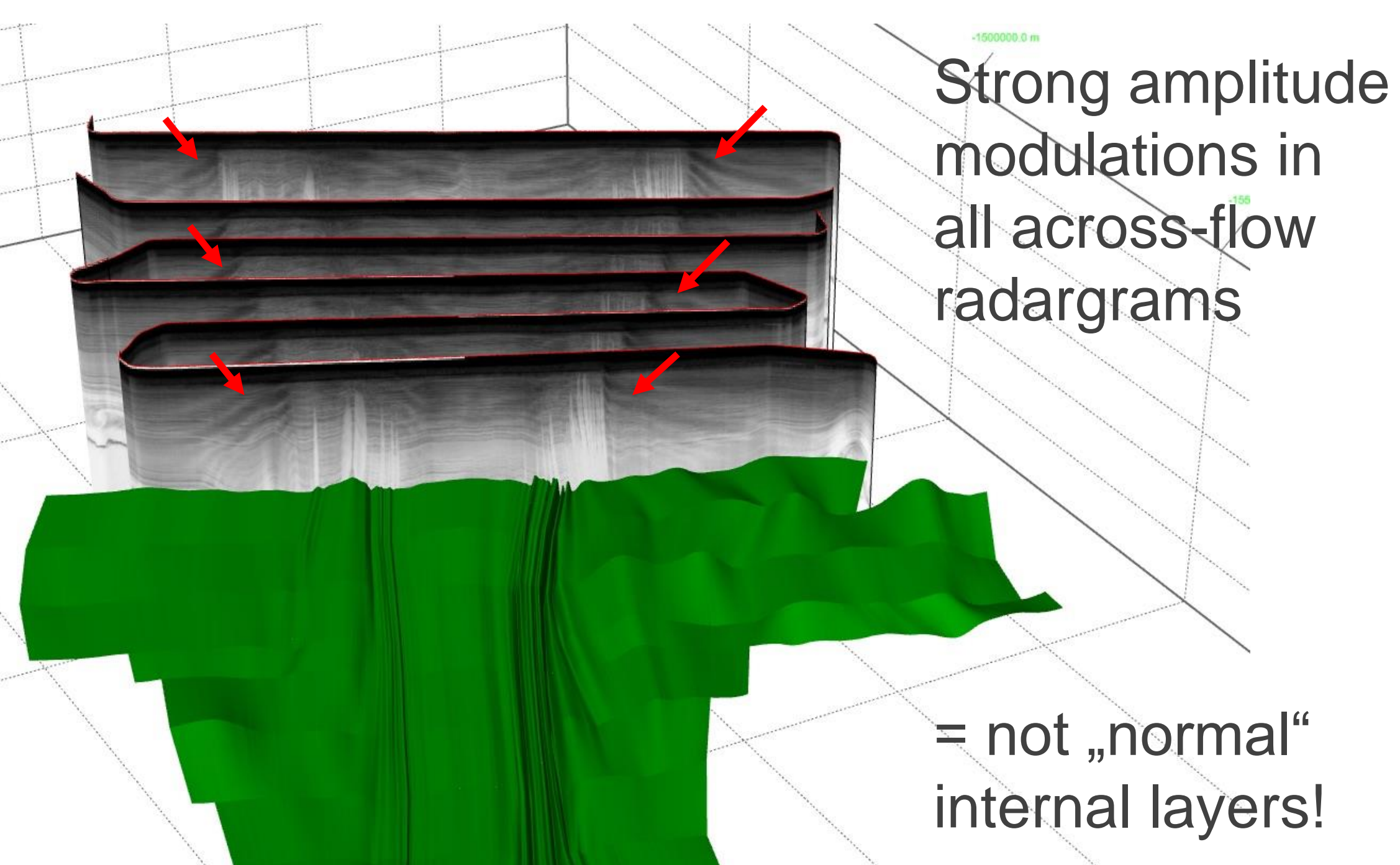




\section{Across flow radargram NEGIS}

amplitude modulation - origin?

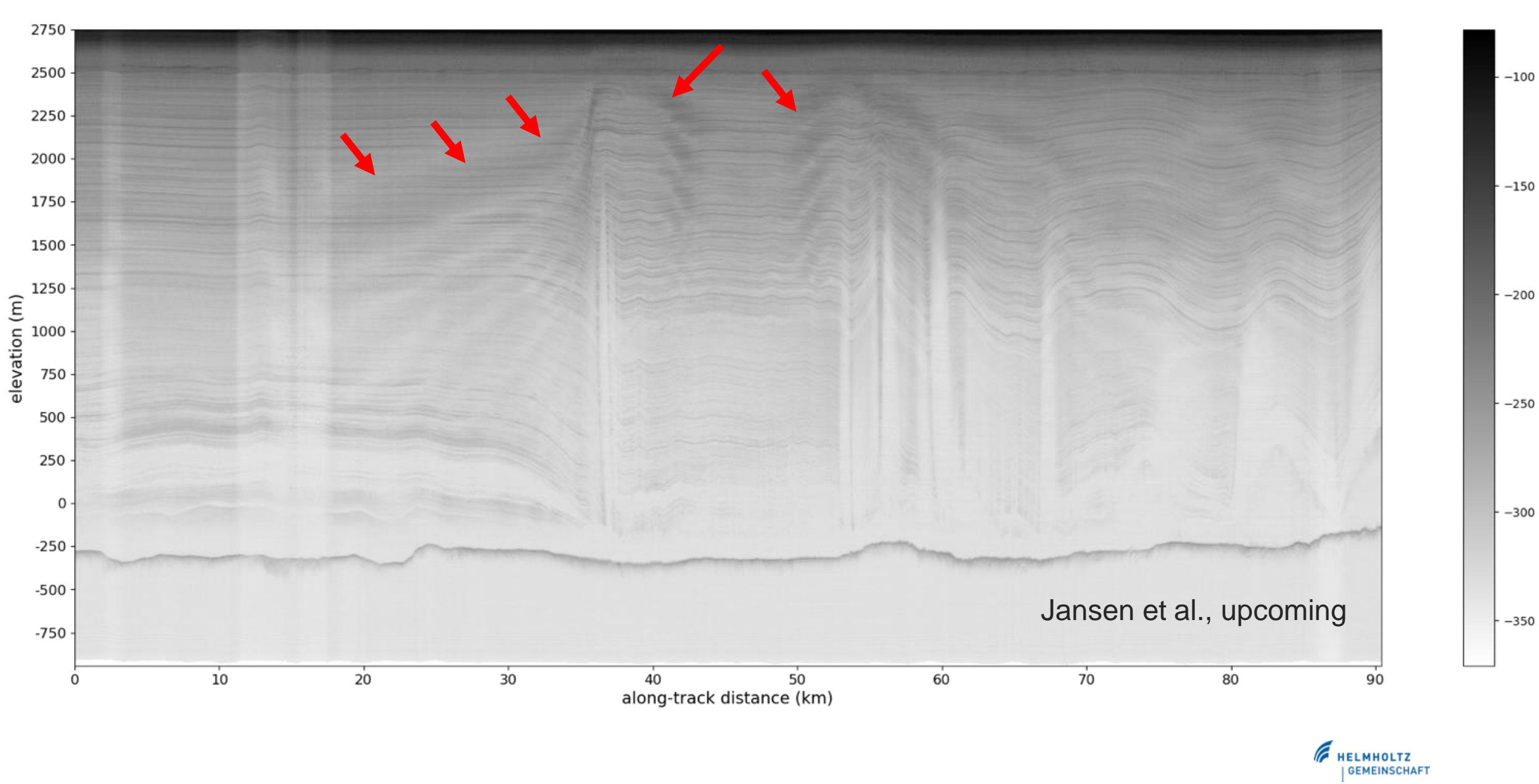




\section{Presentation overview $(=$ 畨)}

\section{Part 1: Physics}

-Where are the modulations coming from?

- What do they tell us?

\section{Part 2: Analysis}

- From observations to horizontal anisotropy

Part 3: Implications

-What can we learn about ice stream dynamics? 


\section{Part 1: Physics - a birefringent medium $\mathbb{C}$ ANI}

ice $1 \mathrm{~h}$ : anisotropic crystal, effects on

rheology ("softness" of ice)

radar velocity:

$$
\begin{gathered}
c=\frac{c_{0}}{\sqrt{\varepsilon^{\prime}}} \\
\varepsilon_{\|}^{\prime}-\varepsilon_{\perp}^{\prime} \approx 1 \% \varepsilon^{\prime}, \quad \varepsilon^{\prime} \approx 3.1-3.2
\end{gathered}
$$

seismic velocity:

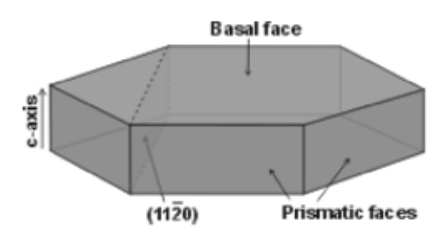

Interference of radar waves

travelling with slightly different

velocities:

$v_{\|}^{s}-v_{\perp}^{s} \approx 100 \mathrm{~ms}^{-1} \approx 5 \% v^{s}, \quad v^{s} \approx 1900 \mathrm{~ms}^{-1}$

$v_{\|}^{p}-v_{\perp}^{p} \approx 100 \mathrm{~ms}^{-1} \approx 3 \% v^{p}, \quad v^{p} \approx 3900 \mathrm{~ms}^{-1}$

$$
\begin{aligned}
\Delta \varepsilon^{\prime} & =\varepsilon_{\|}^{\prime}-\varepsilon_{\perp}^{\prime}=0.034 \\
\Delta \mathrm{c} & =(1.6995-1.6903) 10^{8} \mathrm{~m} / \mathrm{s} \\
& =0.54 \% \mathrm{c}_{0}
\end{aligned}
$$
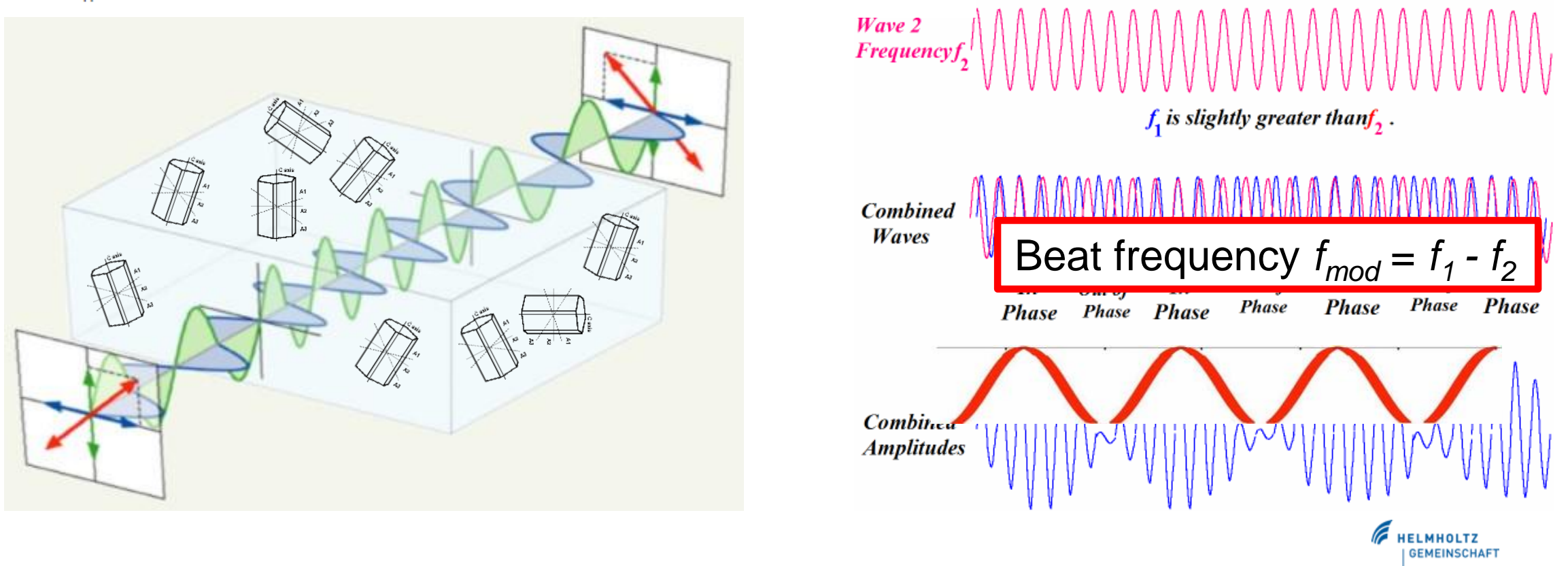


\section{Theory: Fujita et al. (2006) (on ice)}

Bulk properties of polycrystals:

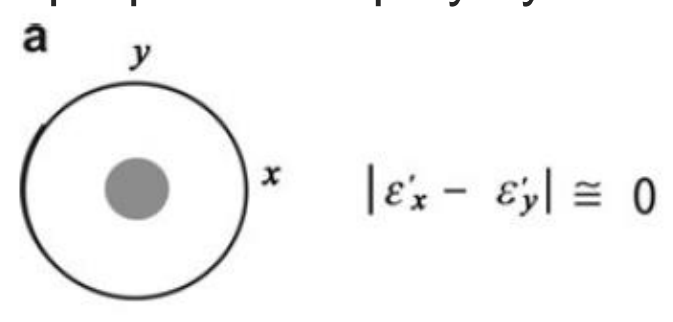

b
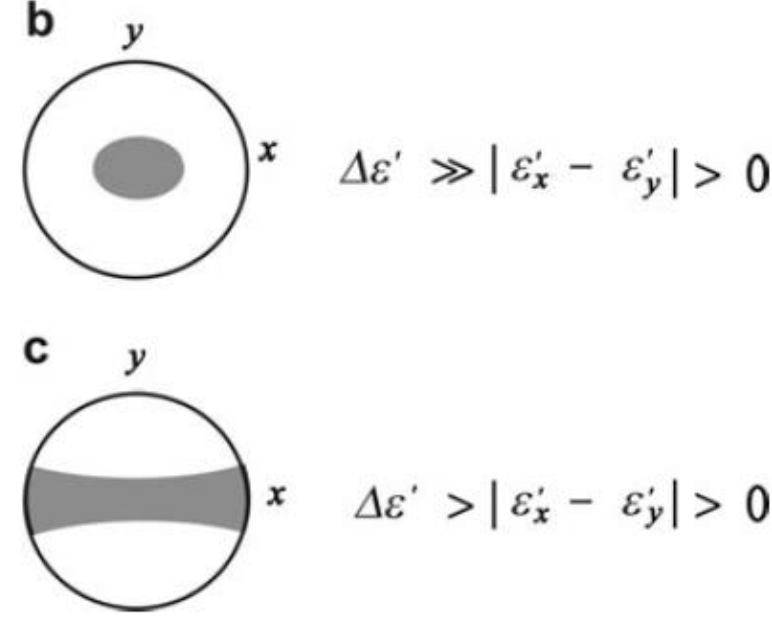

$\Delta \varepsilon^{\prime}>\left|\varepsilon_{x}^{\prime}-\varepsilon_{y}^{\prime}\right|>0$
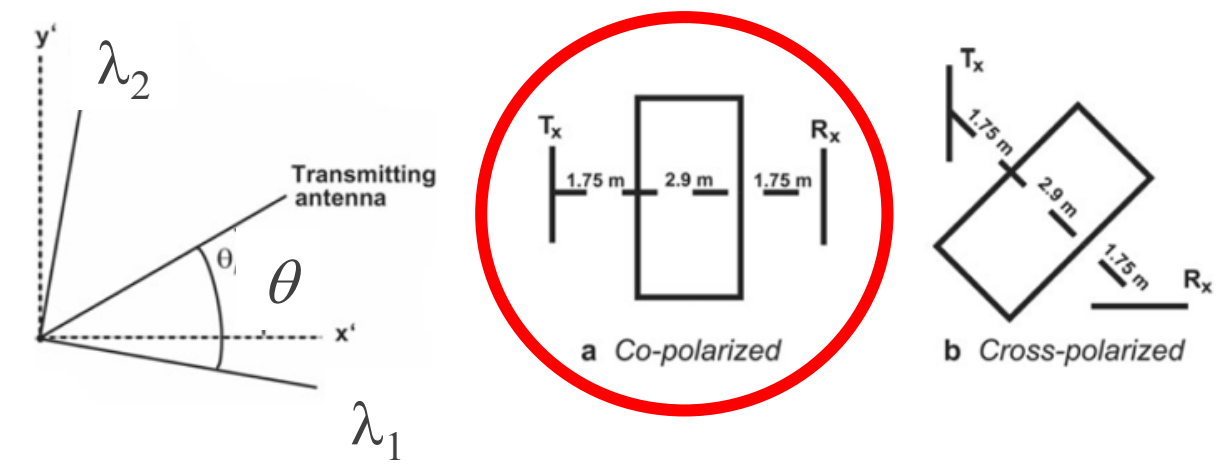

b Cross-polarized

Bulk properties related to single crystals:

$\epsilon^{\prime}(z)=\left(\begin{array}{ccc}\epsilon_{x}^{\prime} & 0 & 0 \\ 0 & \epsilon_{y}^{\prime} & 0 \\ 0 & 0 & \epsilon_{z}^{\prime}\end{array}\right)=\left(\begin{array}{cc}\epsilon_{\perp}^{\prime}+\Delta \epsilon^{\prime} \lambda 1 & 0 \\ 0 & \epsilon_{\perp}^{\prime}+\Delta \epsilon^{\prime} \lambda 2\end{array}\right) \begin{gathered}0 \\ 0 \\ 0\end{gathered}$

$\Delta \varepsilon^{\prime}=\varepsilon_{\|}^{\prime}-\varepsilon_{\perp}^{\prime}=0.034 \quad$ (for a single crystal)

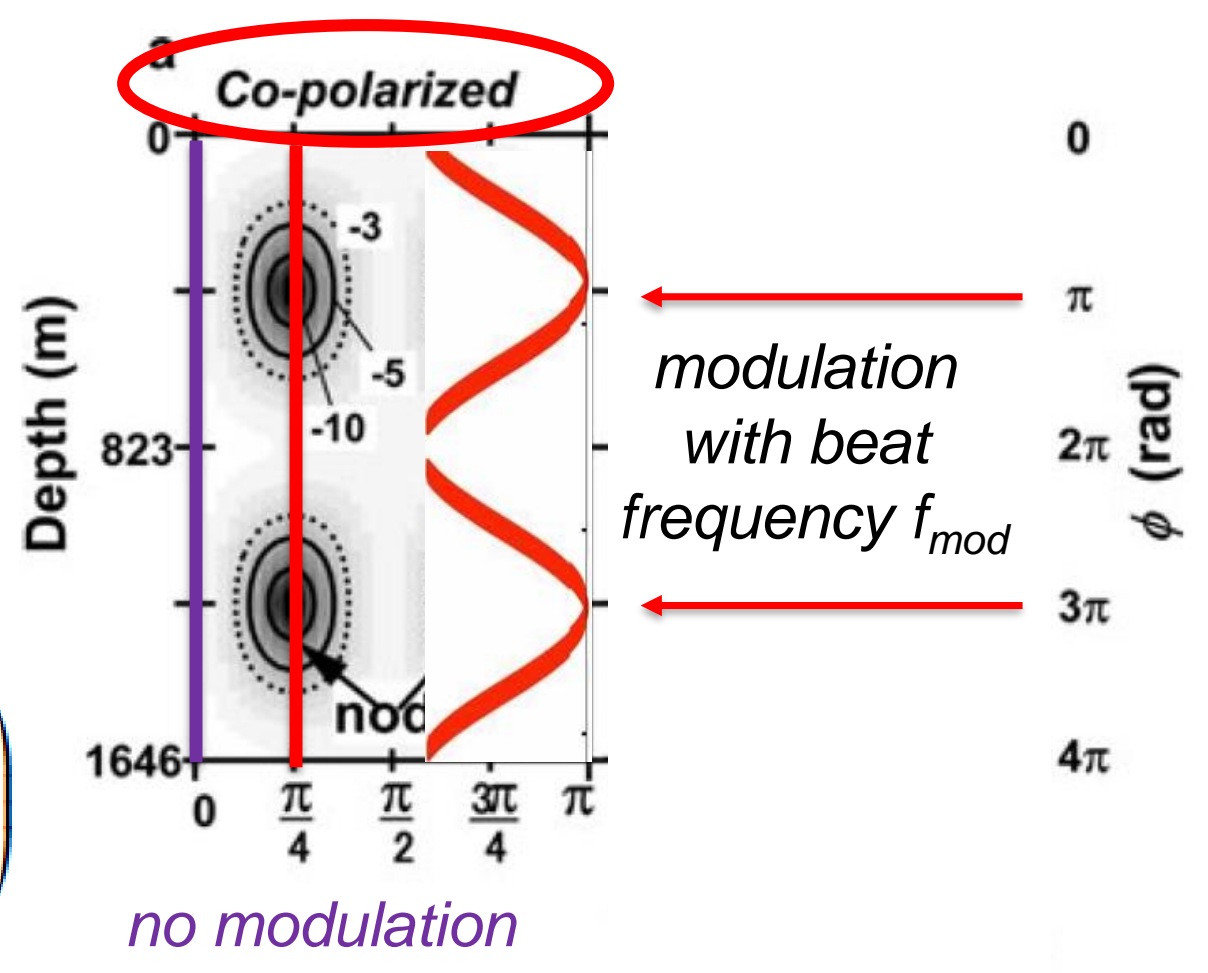




\section{Relating modulation to anisotropy}

Phase difference $\phi$ of ordinary and extraordinary wave cause modulation (Fujita et al., 2006)

$$
\begin{aligned}
& \phi=\frac{4 \pi f}{c_{0}} \int_{z}^{0}\left(\sqrt{\epsilon_{x}^{\prime}}-\sqrt{\epsilon_{y}^{\prime}}\right) d z \\
& =\frac{4 \pi f}{c_{0}} \int_{z}^{0} \frac{\Delta \epsilon(z)}{2 \sqrt{\bar{\epsilon}}} d z \\
& =\text { const } \cdot \Delta \lambda z \quad \text { assuming vertically } \\
& =0.078 \Delta \lambda z \quad \text { for } f=195 \mathrm{MHz} \\
& \begin{aligned}
\Delta \epsilon=\epsilon_{y}-\epsilon_{x} & =\Delta \epsilon^{\prime}\left(\lambda_{2}-\lambda_{1}\right), \\
& =0.034 \Delta \lambda
\end{aligned} \\
& \Sigma_{i} \lambda_{i}=1 \\
& \lambda_{3}=1-2 \lambda_{1}-\Delta \lambda
\end{aligned}
$$

$\Delta \lambda$ : horizontal anisotropy 


\section{Nodes from destructive interference}

- modulation $A \sim \widehat{A_{0}} \cos (\phi)$ : minimum for $\phi=(2 n-1) \pi$

- wave number: $k=2 \pi$ / wavelength $=2 \pi f_{\text {mod }} / c$

forward simulation:

$\phi=k z=0.078 \Delta \lambda z$

$$
\Delta \lambda=0.1 \quad \Delta \lambda=0.2 \quad \Delta \lambda=0.3
$$

$\Delta \lambda \sim k \sim f_{\bmod }$

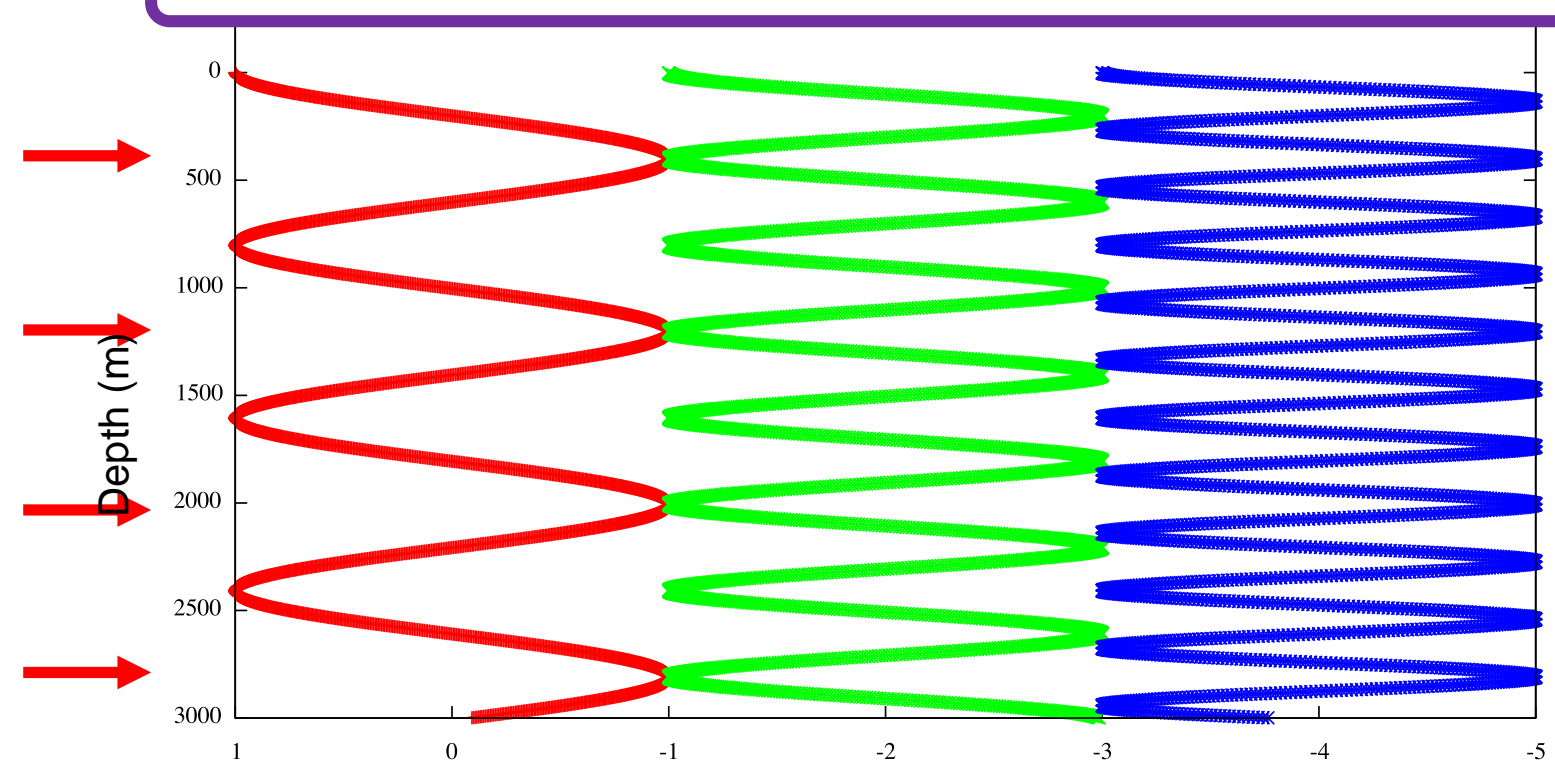

$k / 2 \pi=3.75 / 3000 \mathrm{~m}=7.5 / 3000 \mathrm{~m}=11 / 3000 \mathrm{~m}$

$\begin{array}{cll}=\quad k=0.0078 \mathrm{~m}^{-1} & =0.015 \mathrm{~m}^{-1} & =0.023 \mathrm{~m}^{-1} \\ \Delta \lambda=0.099 & =0.192 & =0.294\end{array}$




\section{What would theory produce?}

increasing $\Delta \lambda\left(\sim k \sim f_{\text {mod }}\right)$

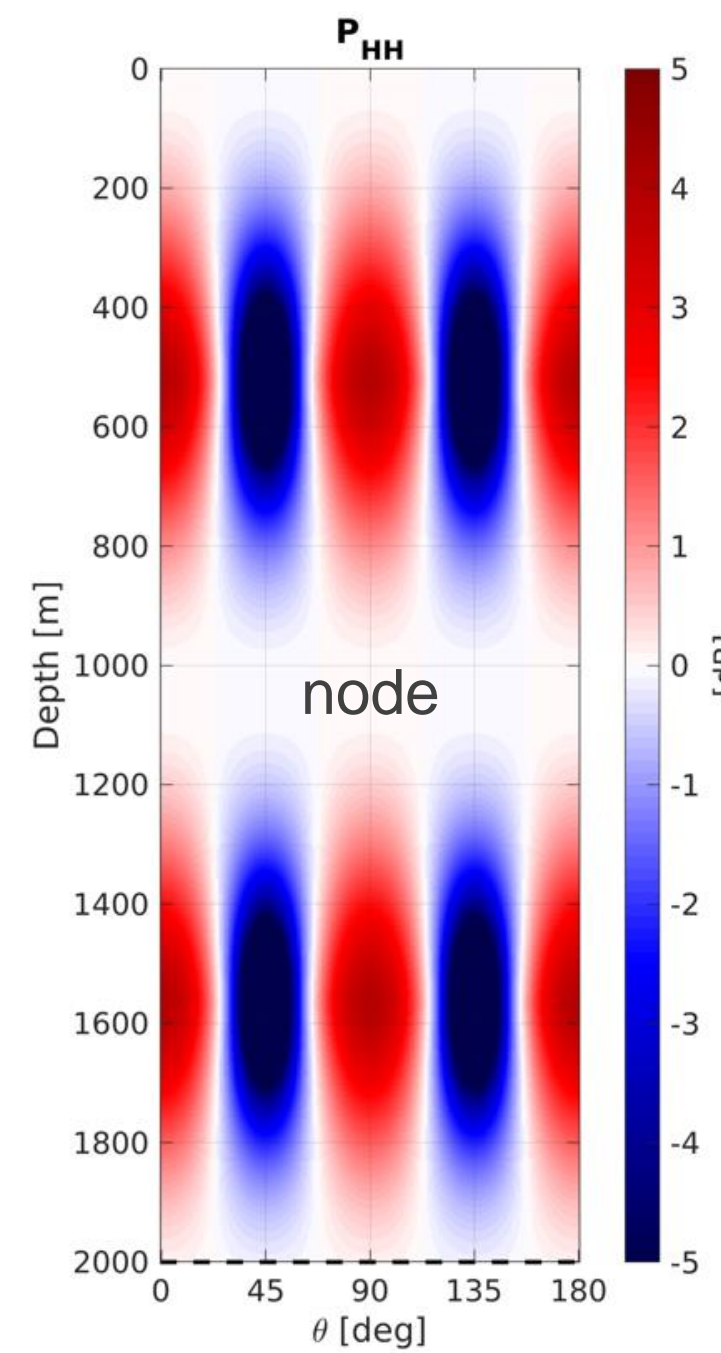

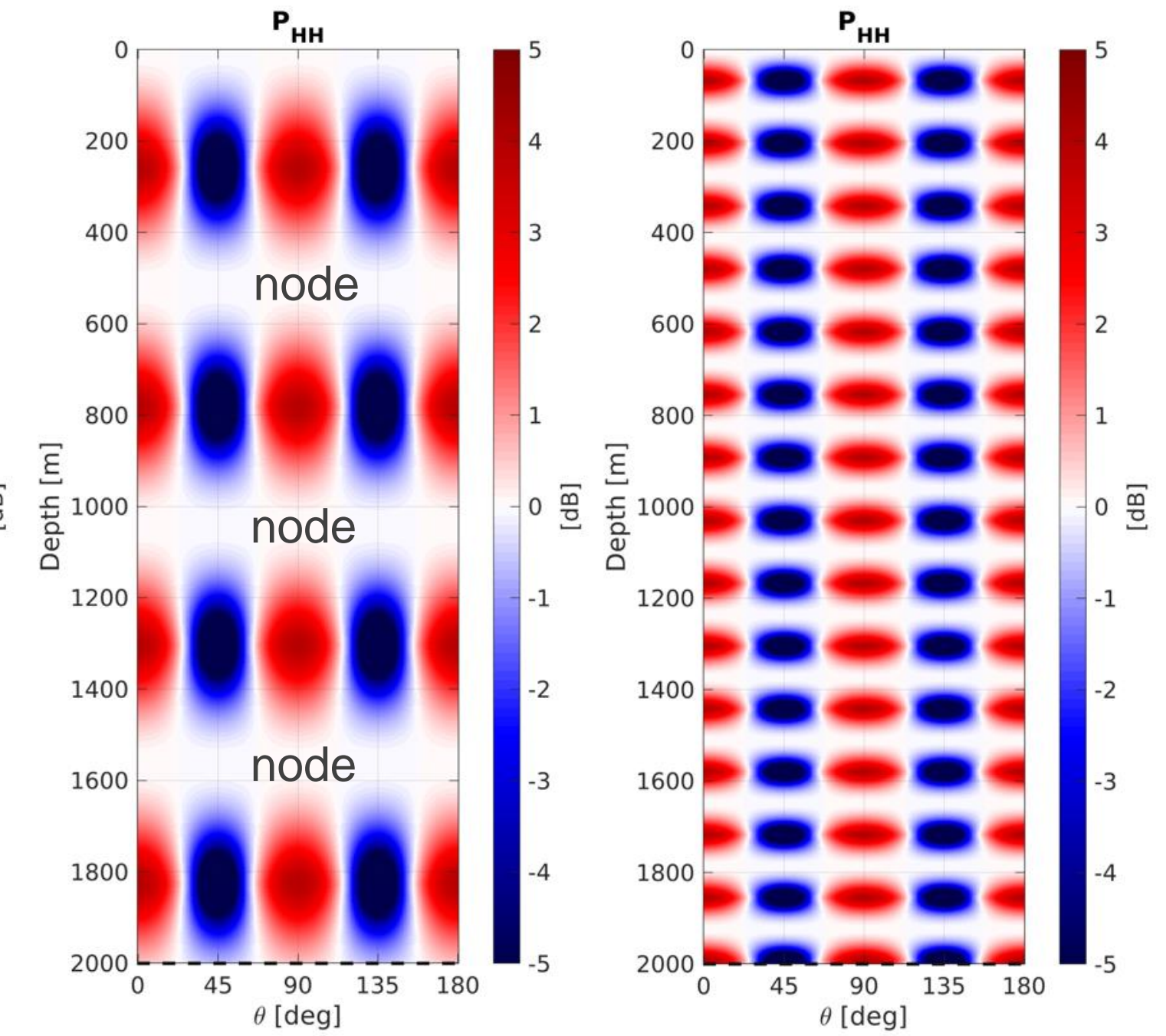

Polarimetric plots (e.g. Ershadi et al., 2021) 


\section{Part 2: Analysis}

\section{Processing flow:}

- pre-processing of radargrams

(remove features which would cause artefacts)

- (semi-)automatic extraction of $f_{\text {mod }}$ from radargrams

(low-pass filtering \& spectrogram analysis)

- mapping of $\Delta \lambda$ along all profiles 


\section{Removing layers to visualize nodes}

Bandpass filtering $\left(f_{\text {mod }}=100-600 \mathrm{kHz} \approx \Delta \lambda=0.03-0.16\right)$

\section{Across:}

Along:
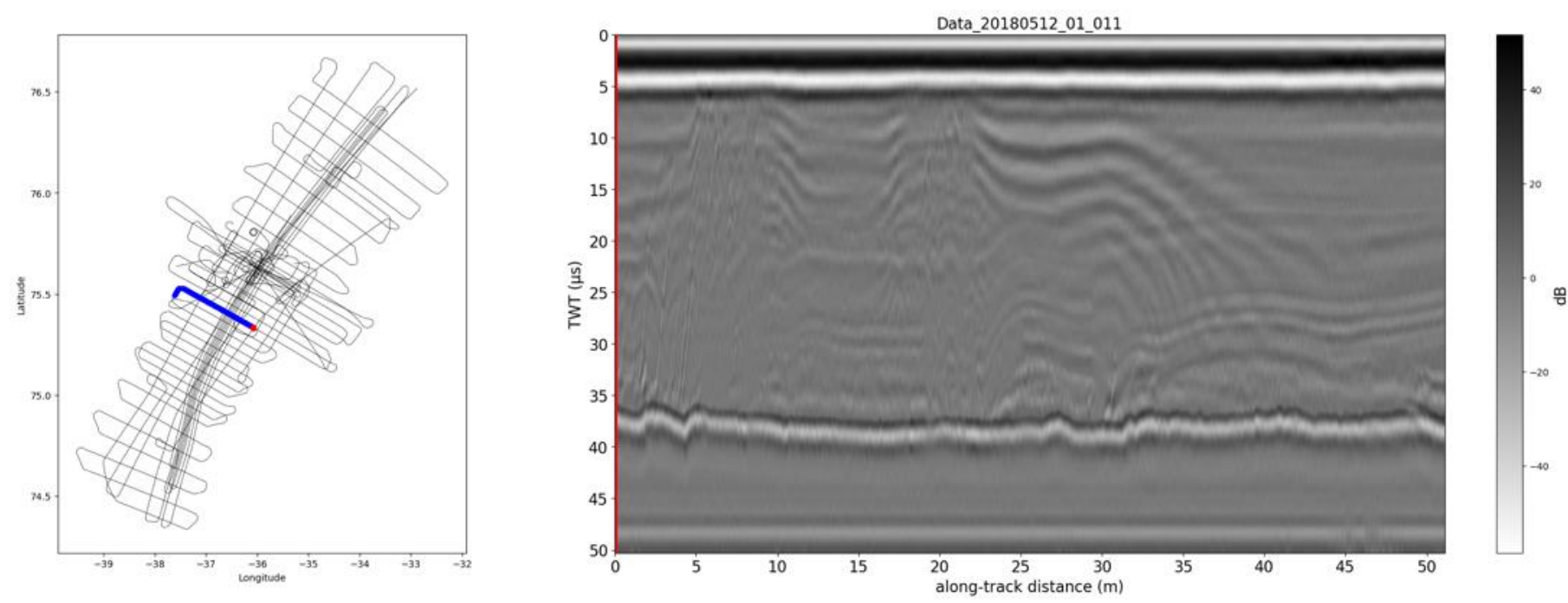

Data_20180510_02_008

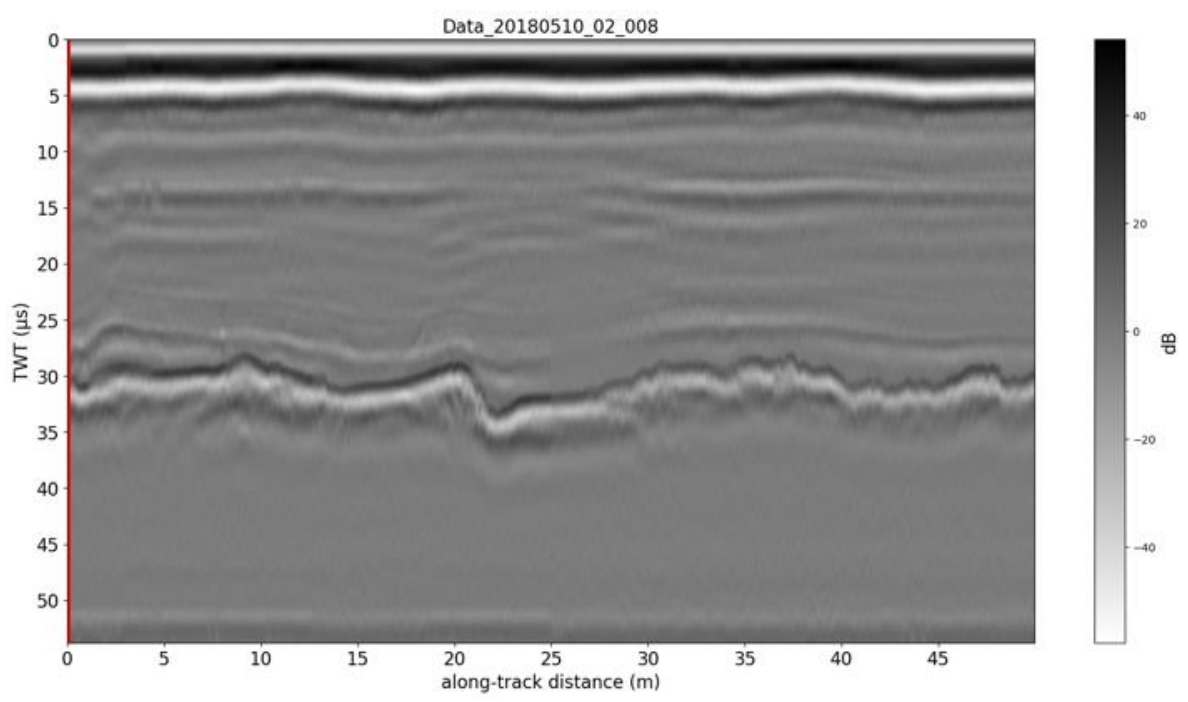




\section{Presence: everywhere

\section{across flow}
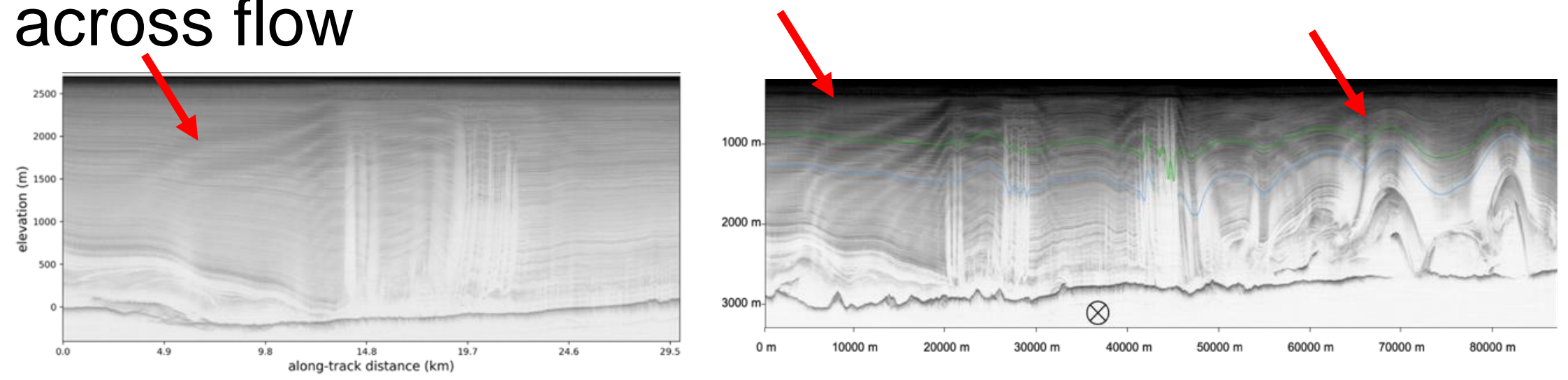

along flow

Jansen et al., upcoming

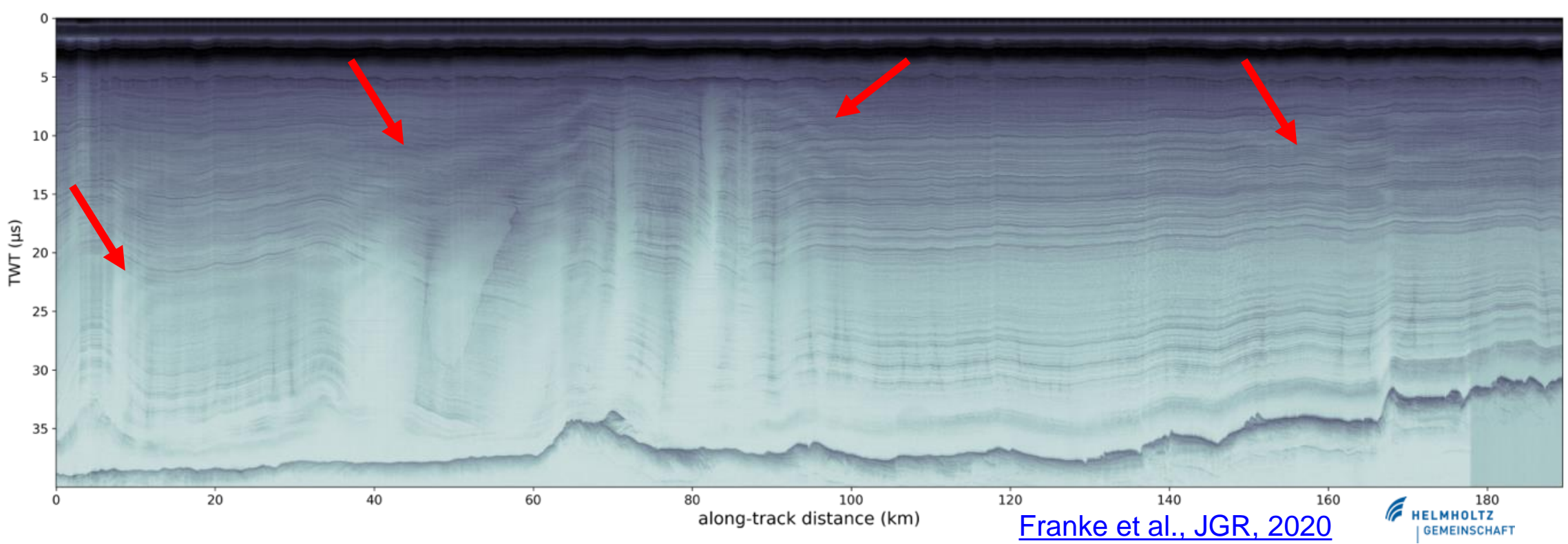




\section{Test: visible in different radar system?}

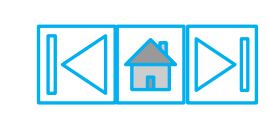

- Ground-based survey 2019

- Mills-T UHF radar, $f=600-900 \mathrm{MHz}$

$=>$ same features, higher $f_{\text {mod }}$ (because of $f$ )
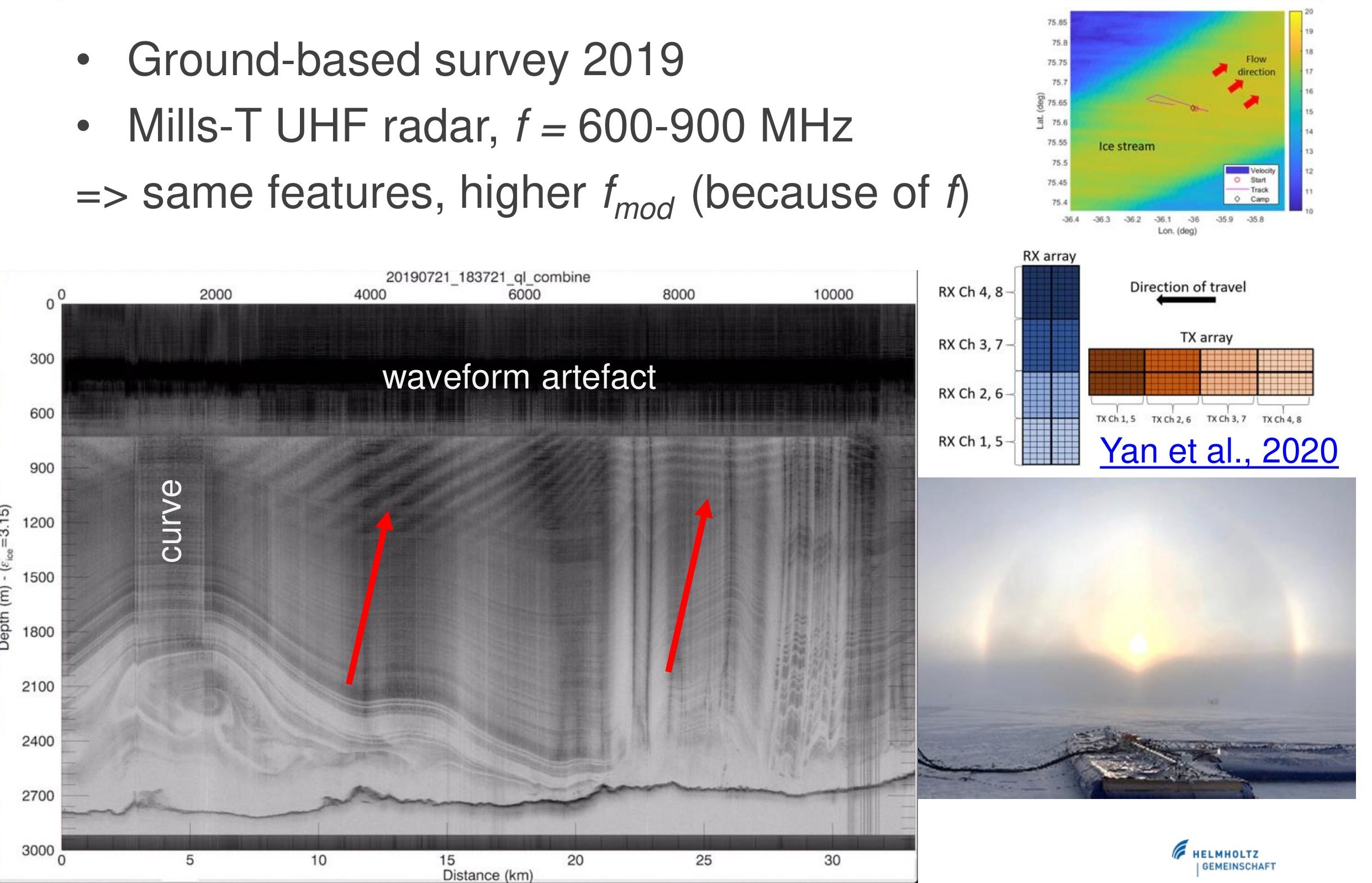


\section{Still not convinced?}

\section{Comparable approach:}

T. J. Young et al.

Polarimetric radar-sounding to infer and quantify shear margin ice fabric anisotropy

EGU21-2107

(Thwaites Glacier eastern shear margin) 


\section{Getting formal: $f_{\text {mod }}$ as $\mathrm{f}(\mathbf{r})$}

\section{Spectrogram along radar profile}

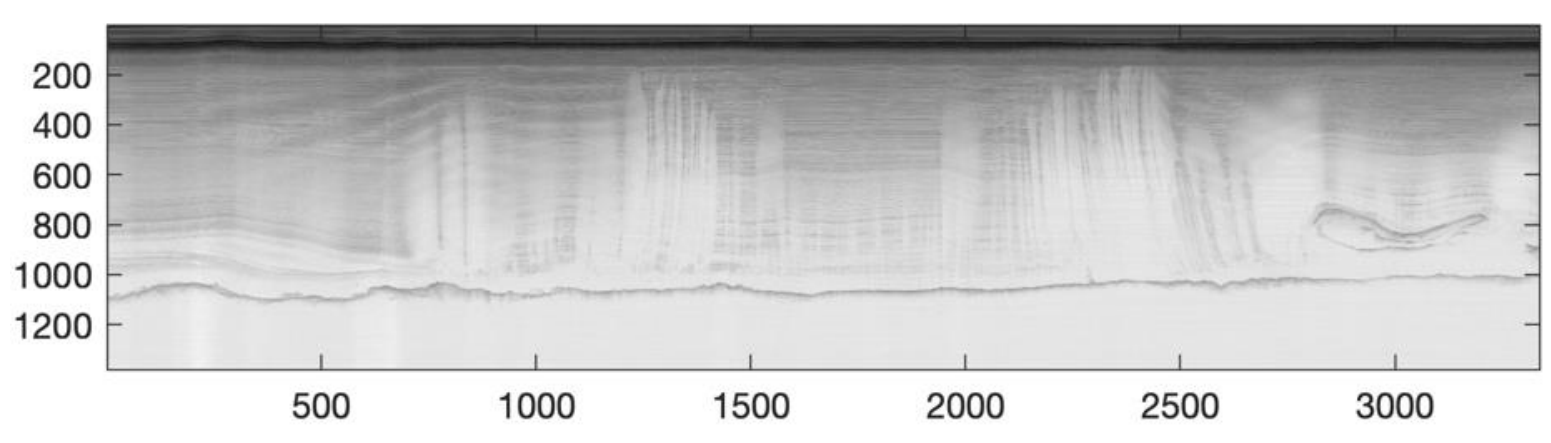

original data

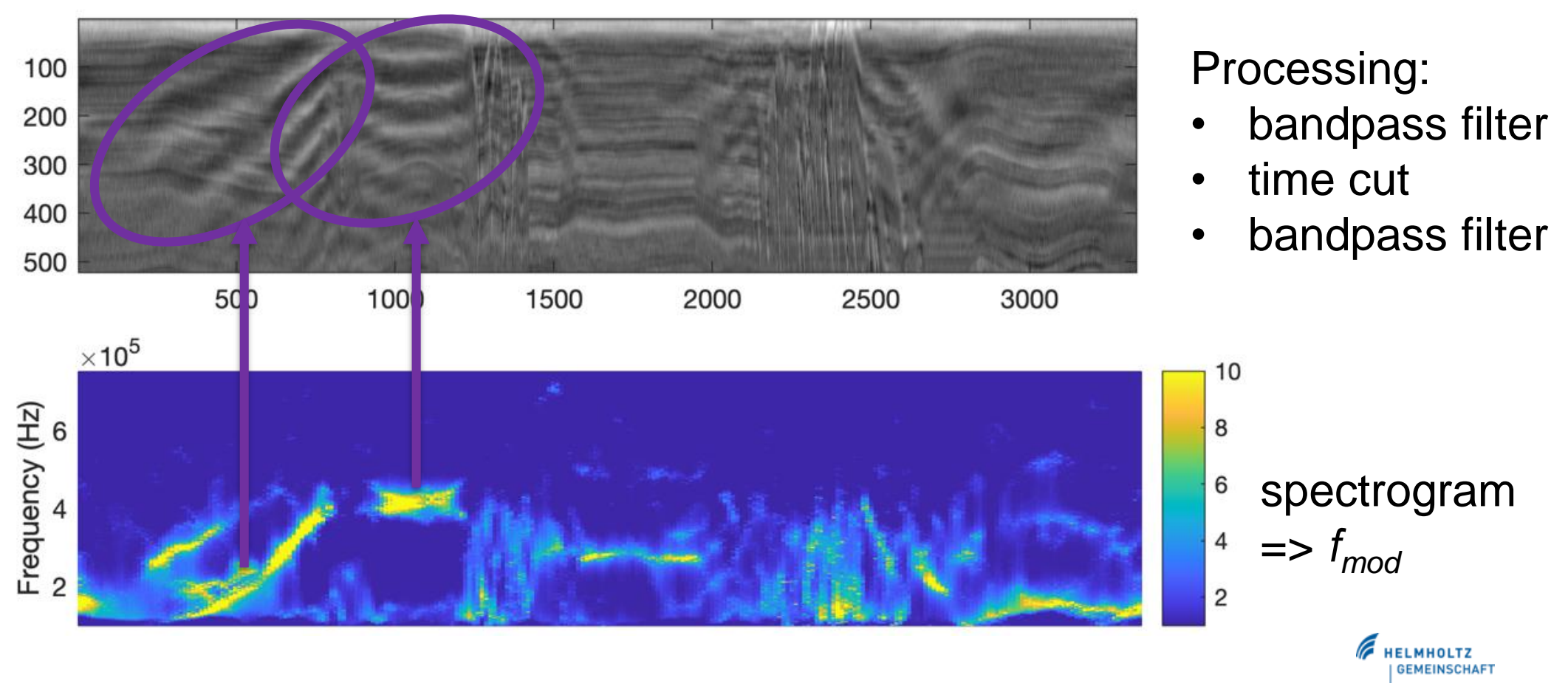




\section{Mapping $\Delta \lambda$}

- displayed: only profiles across flow

- limited range of $\Delta \lambda$

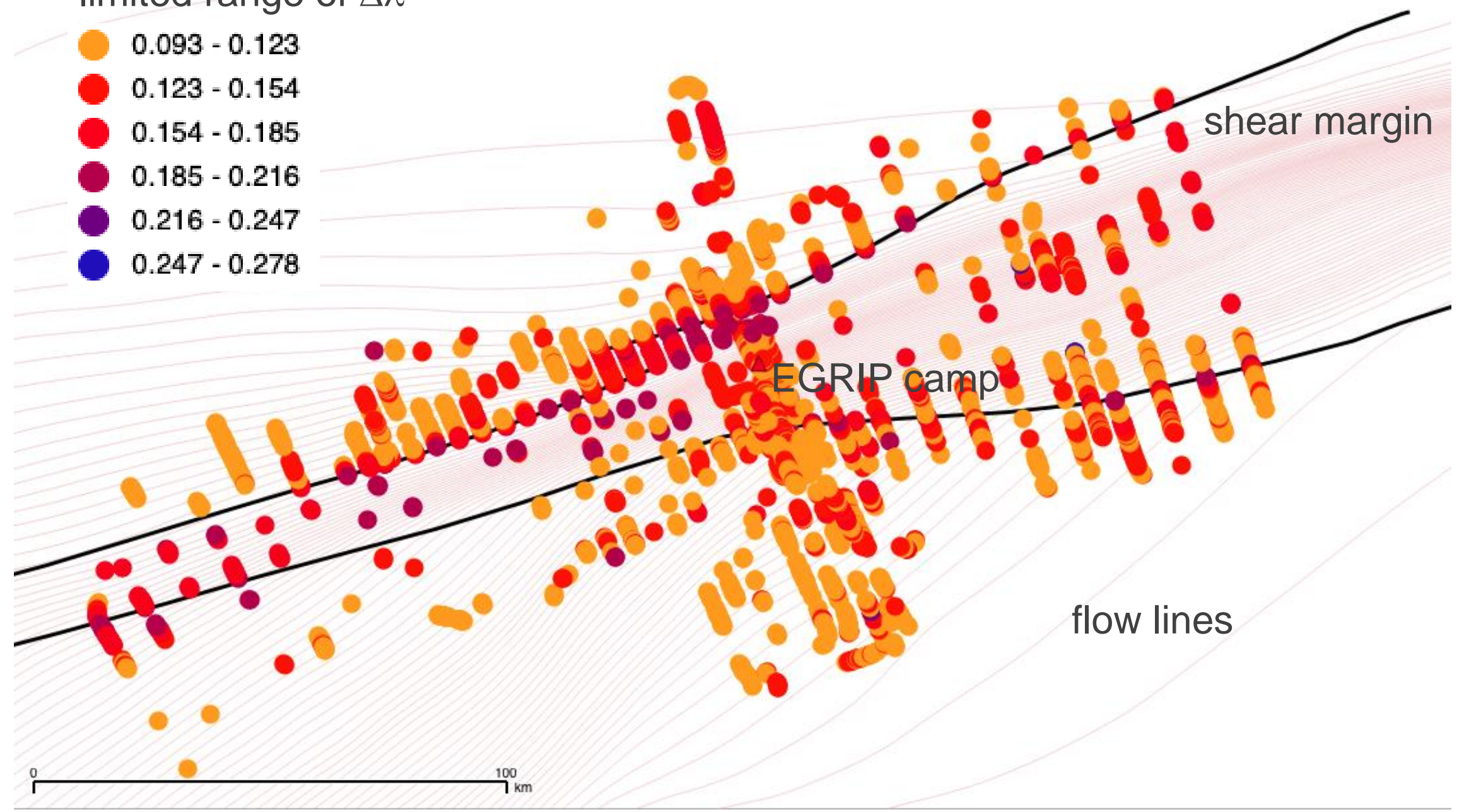




\section{Plausible ranges of $\Delta \lambda(f=195 \mathrm{MHz}$ for AWI UWB) $\mathbb{C}$ AWI}

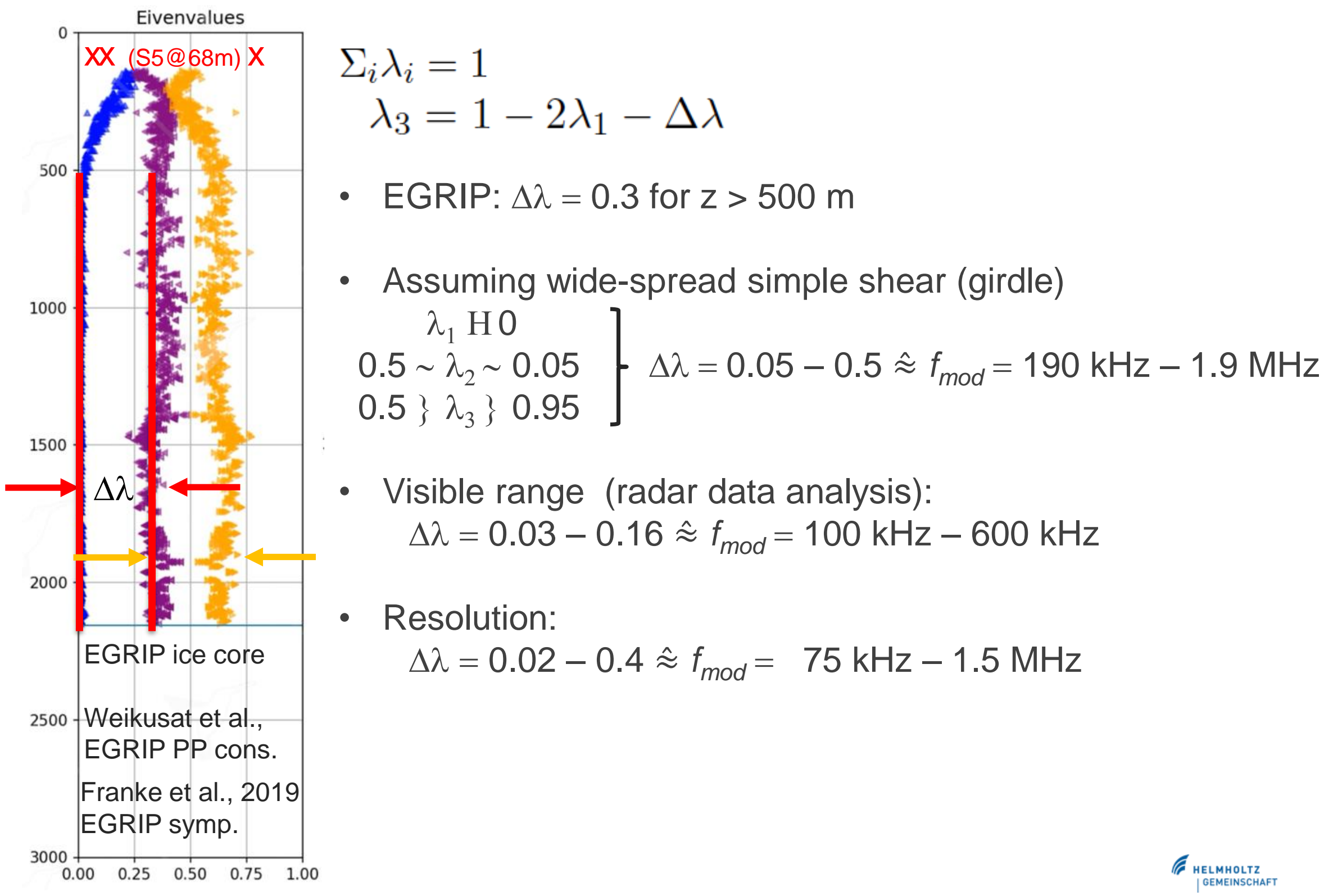




\section{Part 3: Implications}

\section{Questions:}

- What can we actually detect?

-Where do we see horizontal anisotropy?

- How does it change spatially?

- What does it mean for ice dynamics? 


\section{Large-scale flow field}

Strain rates from surface velocities:

- laterally compressive

- accelerating flow

- small-scale variations

- considerable shear 


\section{Dynamics: when does fabric change?

Along-flow signatures of changing fabric anisotropy:

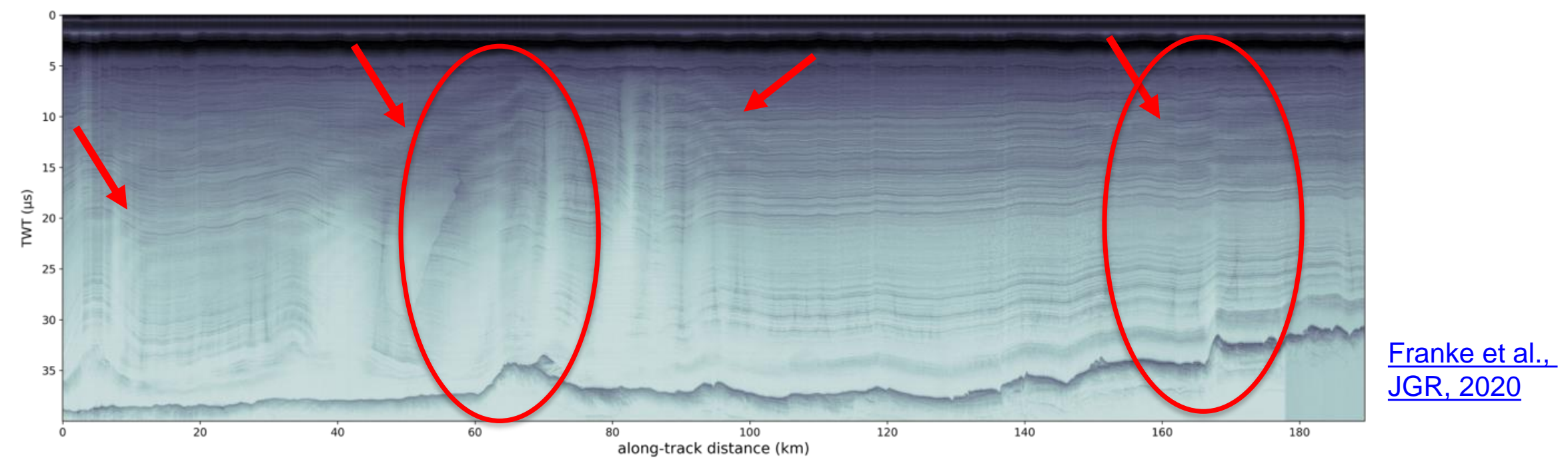

- Horizontal anisotropy seems to change when ice thickness changes

$=>$ Indication of internal deformation?

- Not yet investigated: lateral effects 


\section{NEGIS along-flow profile}

Modelled contribution to flow from internal deformation

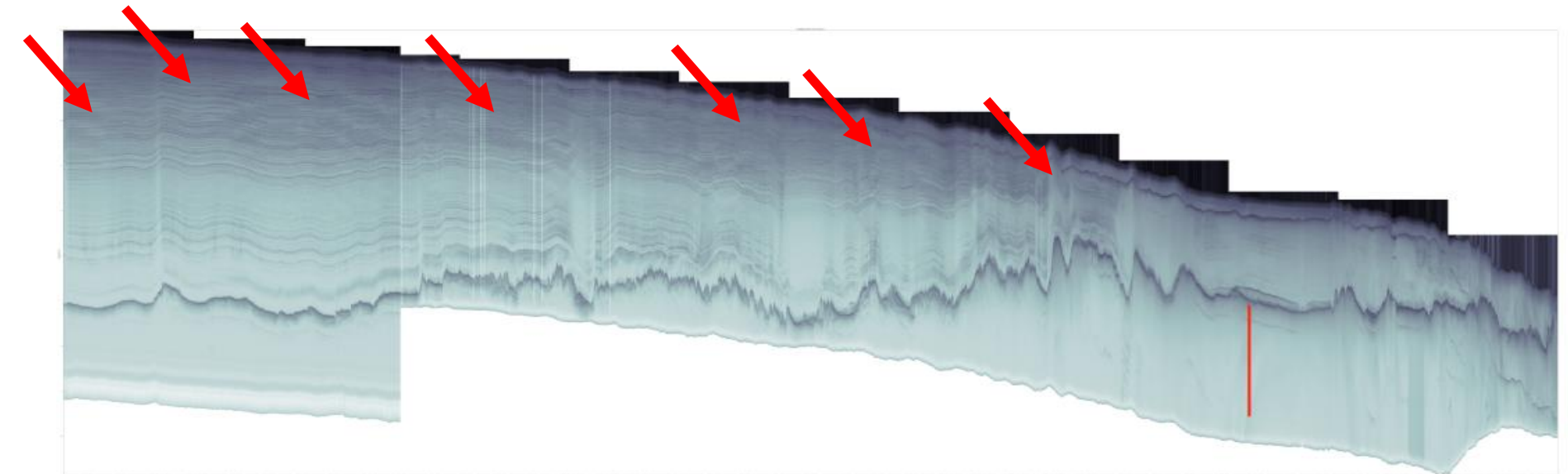

ISSM

$\mathrm{v}$ : velocity

$\mathrm{v}_{\mathrm{b}}$ : basal velocity

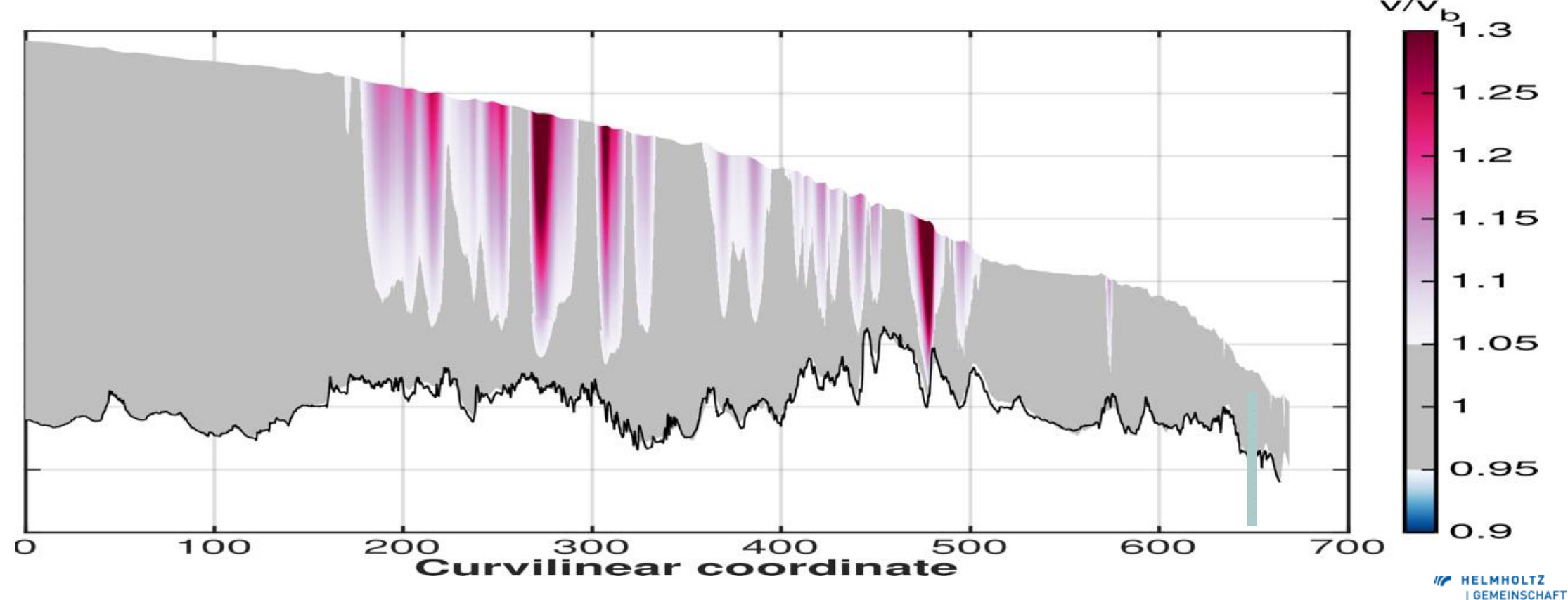

Rückamp/Humbert 


\section{Anisotropic ice-flow model}
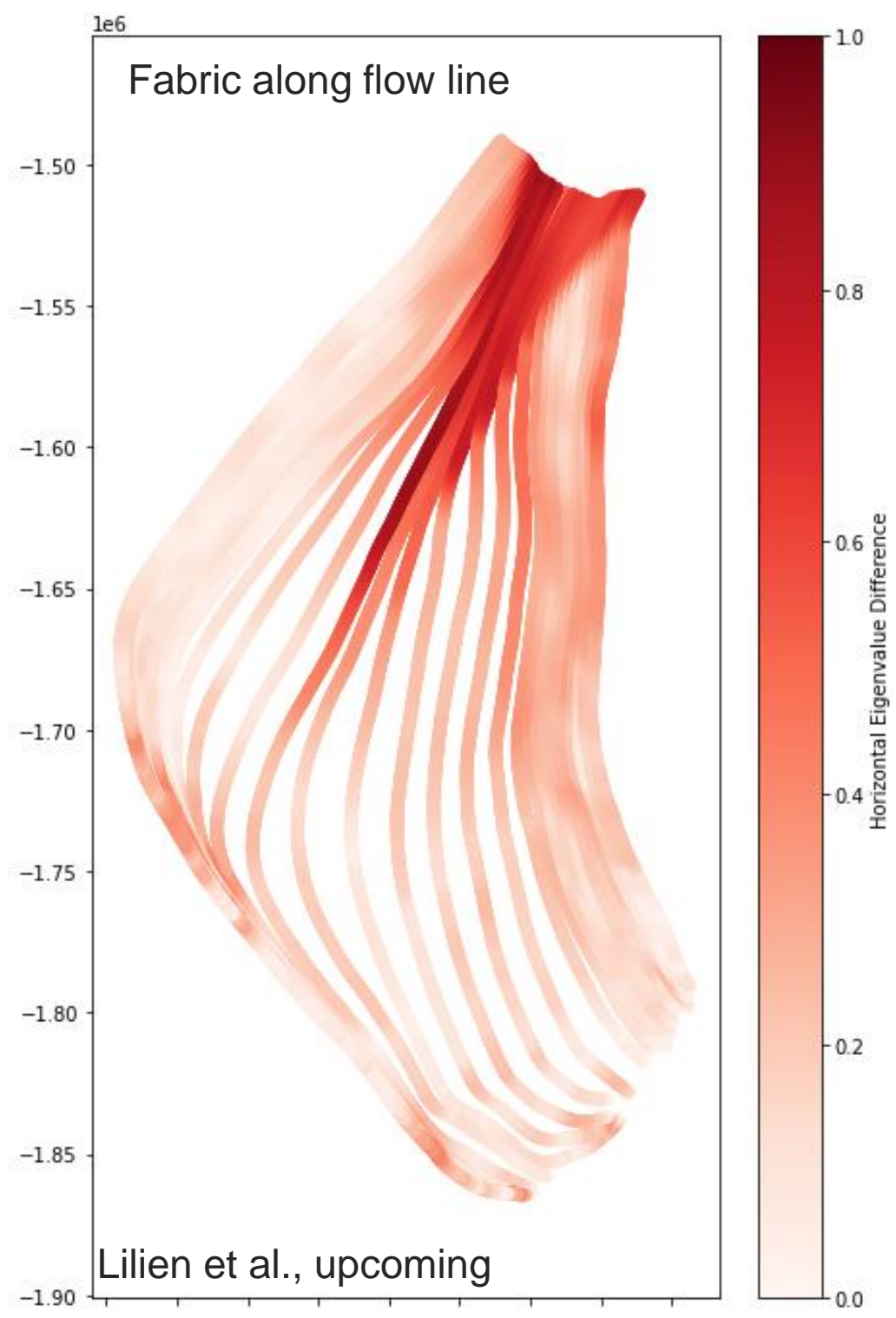

\section{PRELIMINARY!}

\section{ELMER/ICE}

- Bed topography: BedMachine (400 m interpol)

- Anisotropy: GOLF (Gillet-Chaulet et al., 2006)

- No subglacial hydrology

- Basal sliding: linear Weertman
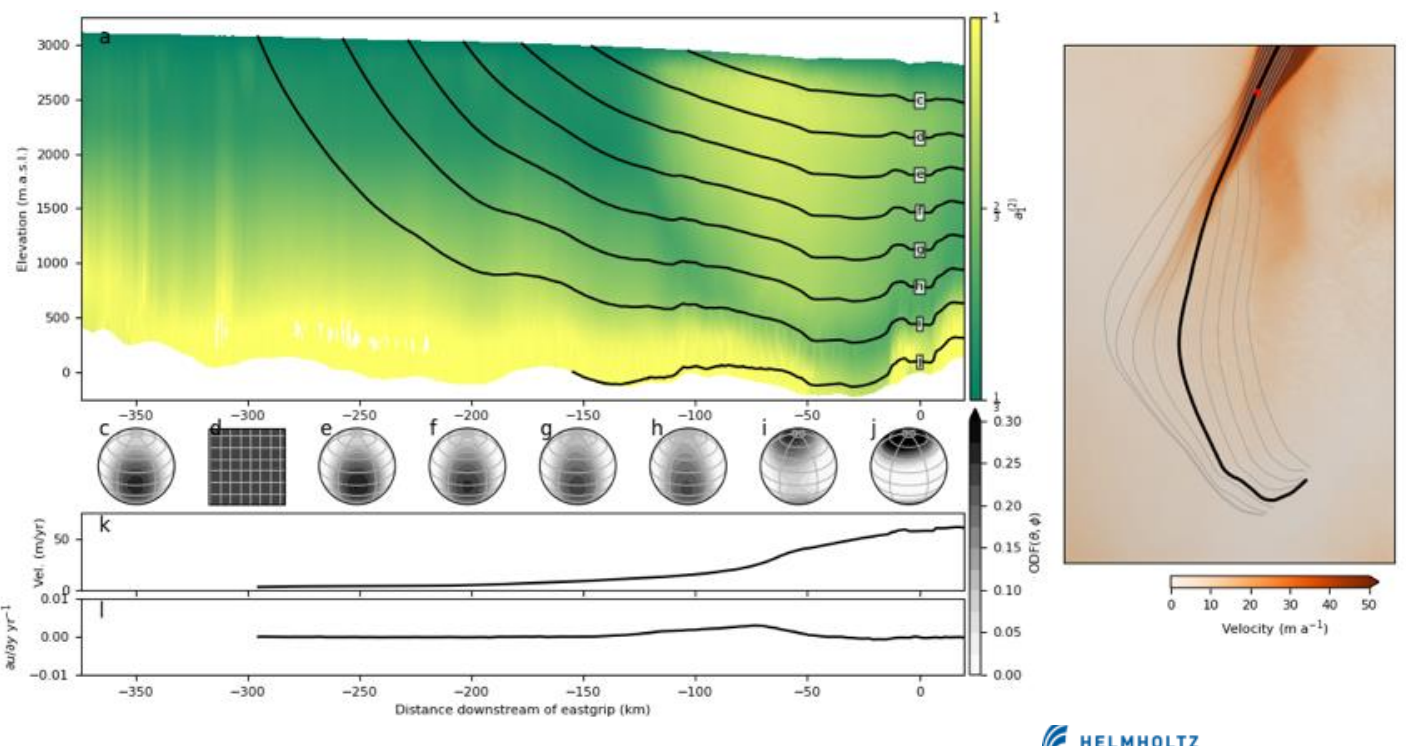


\section{Close-up on northern shear margin
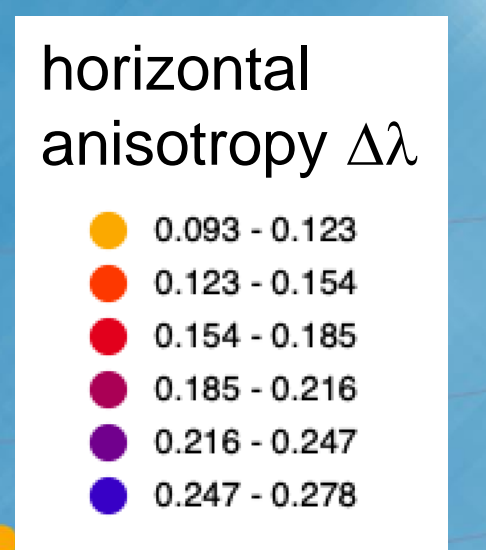


\section{Conclusions I: Methodology}

What do we get from beat frequency?

- strength of horizontal anisotropy $\Delta \lambda=\lambda_{2}-\lambda_{1}$

- as function of position (consistent with ice core)

Disappearing modulation (amplitude $\sim 0$ ):

- orientation of eigenvector || radar profile

- or $\Delta \lambda=0$

Several beat frequencies in spectrogram:

- potentially vertically changing $\Delta \lambda$

\section{Problems:}

- beat signals lost in shear margin (low SNR from folding)

- artefacts in automatic analysis => manual correction 


\section{Conclusions II: Ice-stream dynamics}

Spatial pattern of $\Delta \lambda$ : across flow

- increases towards margin (from outside)

- decreases slightly in ice stream

Spatial pattern of $\Delta \lambda$ : along flow (preliminary)

- $\Delta \lambda$ increases around bedrock undulation

Process interpretation:

- Strong correlation with shear strain rate

- qualitative agreement with anisotropic flow model

- but: mangitude of $\Delta \lambda$ does not match (yet)

Open question:

- how to constrain $\lambda_{3}=1-2 \lambda_{1}-\Delta \lambda$ to get absolute values from co-pol measurements only?

$=>$ non-nadir geometries, other options? 


\section{Acknowledgements}

EastGRIP is directed and organized by the Centre for Ice and Climate at the Niels Bohr Institute, University of Copenhagen. It is supported by funding agencies and institutions in Denmark (A. P. Möller Foundation, University of Copenhagen), USA (US National Science Foundation, Office of Polar Programs), Germany (Alfred Wegener Institute, Helmholtz Centre for Polar and Marine Research), Japan (National Institute of Polar Research and Arctic Challenge for Sustainability), Norway (University of Bergen and Bergen Research Foundation), Switzerland (Swiss National Science Foundation), France (French Polar Institute Paul-Emile Victor, Institute for Geosciences and Environmental research) and China (Chinese Academy of Sciences and Beijing Normal University).

Tobias Binder and the Polar6 crew for data acquisition. Fernando Valero (AWI) for the assisstence regarding automatic processing. 